\title{
Property Analysis of MIMO-Based Missile-Borne Forward-Looking SAR
}

\author{
Yachao Li, Ziqiang Meng, Shengqi Zhu, Yinghui Quan, Mengdao Xing, and Zheng Bao \\ National Laboratory of Radar Signal Processing, Xidian University, Xi'an 710071, China \\ Correspondence should be addressed to Yachao Li; ycli@mail.xidian.edu.cn
}

Received 28 February 2014; Revised 18 June 2014; Accepted 27 June 2014; Published 14 July 2014

Academic Editor: Wei Xu

Copyright (C) 2014 Yachao Li et al. This is an open access article distributed under the Creative Commons Attribution License, which permits unrestricted use, distribution, and reproduction in any medium, provided the original work is properly cited.

\begin{abstract}
As a special multiple-input multiple-output (MIMO) radar networking mode, missile-borne forward-looking synthetic aperture radar (MFL-SAR) has many potential applications. This paper describes and analyzes properties of this new configuration. Range history and Doppler history are analyzed and derived using the designed geometric configuration. Then the expressions of range and Doppler resolution are determined based on the validity of two-dimensional (2D) resolution imaging capability. To help to design the proper system and motion parameters of this configuration, key parameters affecting the imaging ability are found out. Due to high velocities and accelerations of both transmitter and receiver, high-order terms in the slant range equation should be kept to reduce the approximation error. The range resolution and Doppler resolution of MFL-SAR are both space-variant and timevariant owing to the complexity of this configuration. The tiny changes of $2 \mathrm{D}$ resolution during the synthetic aperture time should be considered when designing the imaging algorithm of MFL-SAR.
\end{abstract}

\section{Introduction}

Multiple-input multiple-output (MIMO) [1-4] radar networking system is a new radar mode in which many transmitters synchronize to transmit signals and many other receivers get target echo signals simultaneously. Proposed at the beginning of 21th century, it has been attracting much attention because of its good performance in antistealth, low probability intercept and antijamming capability. Besides, application of MIMO radar networking to radar imaging [5] could improve radar imaging resolution and thus can be further applied to enhance properties of targets detection, recognition, and tracking $[6,7]$. As one special MIMO radar networking mode, missile-borne multistatic synthetic aperture radar (SAR) has potential applications on ground detection, battlefield reconnaissance, active homing guidance, and so forth. Electromagnetic wave transmitted by many transmitters impinges receivers after reflecting from target area and gets centralized processing $[8,9]$. Such technology can achieve high-resolution imaging of high speed platform for those targets in the straight-ahead position and realize tracking and attacking with high precision, so it can be used in terminal guidance of missile $[10,11]$ especially.
In order to confirm the validity of application of such MIMO radar networking system to missile-borne platform, the mode of "single-transmitting and single-receiving" is assumed, that is, missile-borne bistatic forward-looking SAR (MBFL-SAR). MBFL-SAR is a new bistatic SAR imaging mode [12], which has advantage of $2 \mathrm{D}$ imaging ability in the forward-looking mode over monostatic SAR [13]. Owing to the "far-transmitting and near-receiving" mode and forwardlooking mode of such configuration, transmitter can be placed in safe area far away from target area, while attack missile receives target echo signals in electromagnet silence state, acting as receiver. MBFL-SAR can work to image and seek targets actively during the whole terminal attacking stage because of its low probability intercept and good stealth performance. As one mode of bistatic SARs, MBFL-SAR is also subject to the problems and special requirements of synchronization of the transmitter and receiver. Some investigations were conducted and appealing approaches were suggested on synchronization of bistatic SAR [1416]. This paper concentrates on description and analysis of the properties of this new configuration and ignores the synchronization problem. 
Configuration and resolution analysis are the basis of MBFL-SAR imaging study, and some researches have been published. Three different bistatic forward-looking SAR configurations have been analyzed, and the optimal geometry configurations have been proposed in [17]. Qiu and $\mathrm{Hu}$ designed bistatic forward-looking geometry with stationary transmitter and forward-looking receiver and analyzed its $2 \mathrm{D}$ resolution in [18]. Experiments have already been carried out to test the bistatic forward-looking imaging ability [19, 20]. Some imaging algorithms of bistatic forward-looking configuration have been suggested [21-23]. But all investigations above are focused on airborne or space-borne bistatic forward-looking configurations; a few studies about missileborne platform have been achieved.

In this paper, we describe and analyze this special configuration. Geometric configuration and signal model of MBFL-SAR are introduced in Section 2. High-order terms appearing in the range equation cannot be ignored, because of the fact that both transmitter and receiver have high velocities and accelerations. Doppler parameters are found out and analyzed based on the Taylor series expansion of the range equation. In Section 3, we give the derivation of $2 \mathrm{D}$ resolution of MBFL-SAR and find out key parameters affecting the imaging performance. Several examples are provided to illustrate the resolution ability in the MBFL-SAR in Section 4. Finally, conclusions are drawn in Section 5.

\section{Geometric Configuration and Signal Model}

2.1. Geometric Configuration. MBFL-SAR configuration is shown in Figure 1, in which both transmitter and receiver travel in curvilinear descending motion. $P\left(x_{p}, y_{p}, 0\right)$ (here, the height of the scene is assumed to be zero) is a target point at any position of the dashed area, that is, the imaging area. Transmitter is descending along the curve $\widehat{A}_{t} B_{t}$ in the plane which forms an included angle $\psi$ with plane $y \mathrm{O} z$, in which receiver is travelling along $\widehat{A_{r} B_{r}}$. $O$ is the origin of coordinates, and $O^{\prime}$ is the projection of transmitter when the slow time $t_{m}=0$. For convenience of description, all motion parameters of transmitter are described in coordinates $x^{\prime} O^{\prime} y^{\prime} z$, and the ones of receiver are described in coordinates $x \mathrm{O} y z$. Assume that, at $t_{m}=0$, transmitter and receiver are at $T_{0}\left(x_{t}^{\prime}, 0, H_{T}\right)$ and $R_{0}\left(0,0, H_{R}\right)$, respectively. Velocity vectors of transmitter and receiver at that moment are $\vec{v}_{r 0}=\left(0, v_{r y 0}, v_{r z 0}\right)$ and $\vec{v}_{t 0}=\left(0, v_{t y^{\prime} 0}, v_{t z 0}\right)$, respectively; acceleration vectors are $\vec{a}_{r}=\left(0, a_{r y}, a_{r z}\right)$ and $\vec{a}_{t}=\left(0, a_{t y^{\prime}}, a_{t z}\right)$, respectively. The components in directions $y^{\prime}$ and $z$ are $v_{t y^{\prime}}$ and $v_{t z}$, respectively; the components in directions $y$ and $z$ are $v_{r y}$ and $v_{r z}$, respectively.

The locations of transmitter and receiver at $t_{m}$ are $\left(0, y_{r}, z_{r}\right)$ and $\left(x_{t}^{\prime}, y_{t}^{\prime}, z_{t}\right)$; then we have

$$
\begin{aligned}
& \left(0, y_{r}, z_{r}\right) \\
& \quad=\left(0, v_{r y 0} t_{m}+0.5 a_{r y} t_{m}^{2}, H_{R}+v_{r z 0} t_{m}+0.5 a_{r z} t_{m}^{2}\right), \\
& \left(x_{t}^{\prime}, y_{t}^{\prime}, z_{t}\right) \\
& \quad=\left(x_{t}^{\prime}, v_{t y^{\prime} 0} t_{m}+0.5 a_{t y^{\prime}} t_{m}^{2}, H_{T}+v_{t z 0} t_{m}+0.5 a_{t z} t_{m}^{2}\right) .
\end{aligned}
$$

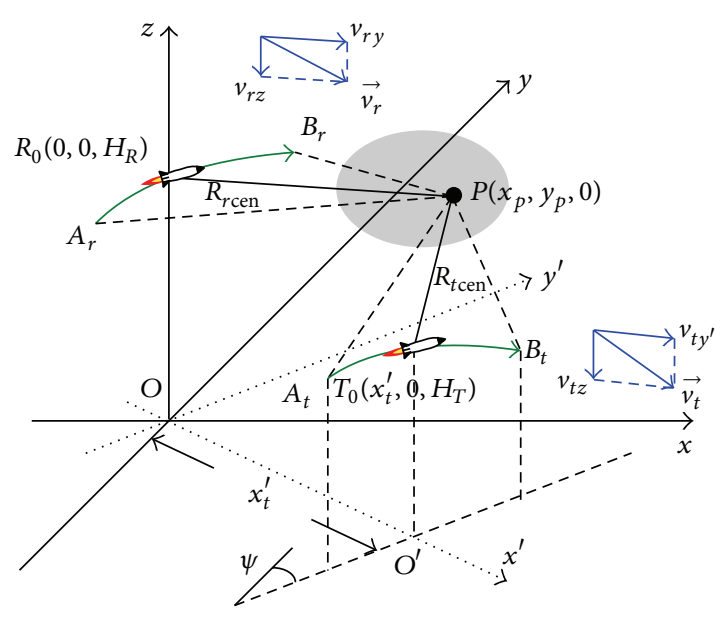

FIGURE 1: Geometric configuration of MBFL-SAR.

The position of transmitter $\left(x_{t}^{\prime}, y_{t}^{\prime}, z_{t}\right)$ in coordinates $x \mathrm{O} y z$, according to the rotation relationship between coordinates $x \mathrm{O} y z$ and $x^{\prime} \mathrm{O} y^{\prime} z$, can be expressed as

$$
\left(x_{t}, y_{t}, z_{t}\right)=\left(x_{t}^{\prime} \cos \psi+y_{t}^{\prime} \sin \psi,-x_{t}^{\prime} \sin \psi+y_{t}^{\prime} \cos \psi, z_{t}\right) .
$$

Then we can obtain the expression of instantaneous bistatic slant range as

$$
\begin{aligned}
R_{\mathrm{bf}}\left(t_{m}\right)= & R_{R}\left(t_{m}\right)+R_{T}\left(t_{m}\right) \\
= & \left(x_{p}^{2}+\left(v_{r y 0} t_{m}+0.5 a_{r y} t_{m}^{2}-y_{p}\right)^{2}\right. \\
& \left.+\left(H_{R}+v_{r z 0} t_{m}+0.5 a_{r z} t_{m}^{2}\right)^{2}\right)^{1 / 2} \\
+ & \left(\left(x_{t}^{\prime}-x_{p}^{\prime}\right)^{2}+\left(v_{t y^{\prime} 0} t_{m}+0.5 a_{t y^{\prime}} t_{m}^{2}-y_{p}^{\prime}\right)^{2}\right. \\
& \left.+\left(H_{T}+v_{t z 0} t_{m}+0.5 a_{t z} t_{m}^{2}\right)^{2}\right)^{1 / 2} \\
= & \sqrt{R_{r c e n}^{2}+\mu_{r 1} t_{m}+\mu_{r 2} t_{m}^{2}+\mu_{r 3} t_{m}^{3}+\mu_{r 4} t_{m}^{4}} \\
& +\sqrt{R_{t c e n}^{2}+\mu_{t 1} t_{m}+\mu_{t 2} t_{m}^{2}+\mu_{t 3} t_{m}^{3}+\mu_{t 4} t_{m}^{4}}
\end{aligned}
$$

where $\left(x_{p}^{\prime}, y_{p}^{\prime}, 0\right)$ is the position of $\left(x_{p}, y_{p}, 0\right)$ in coordinates $x^{\prime} O y^{\prime} z$; it can be obtained using the method similar to (2) and with

$$
\begin{gathered}
R_{r \text { cen }}=\sqrt{x_{p}^{2}+y_{p}^{2}+H_{R}^{2}}, \\
R_{t \text { cen }}=\sqrt{\left(x_{t}^{\prime}-x_{p}^{\prime}\right)^{2}+y_{p}^{\prime 2}+H_{T}^{2}}, \\
\mu_{r 1}=-2 v_{r y 0} y_{p}+2 v_{r z 0} H_{R}, \quad \mu_{t 1}=-2 v_{t y^{\prime} 0} y_{p}^{\prime}+2 v_{t z 0} H_{T}, \\
\mu_{r 2}=v_{r y 0}^{2}+v_{r z 0}^{2}-a_{r y} y_{p}+a_{r z} H_{R},
\end{gathered}
$$




$$
\begin{gathered}
\mu_{t 2}=v_{t y^{\prime} 0}^{2}+v_{t z 0}^{2}-a_{t y^{\prime}} y_{p}^{\prime}+a_{t z} H_{T}, \\
\mu_{r 3}=a_{r y} v_{r y 0}+a_{r z} v_{r z 0}, \quad \mu_{t 3}=a_{t y^{\prime}} v_{t y^{\prime} 0}+a_{t z} v_{t z 0}, \\
\mu_{r 4}=\frac{1}{4}\left(a_{r y}^{2}+a_{r z}^{2}\right), \quad \mu_{t 4}=\frac{1}{4}\left(a_{t y^{\prime}}^{2}+a_{t z}^{2}\right) .
\end{gathered}
$$

2.2. Signal Model. Suppose that the transmitted waveform is the linear frequency modulation (LFM), and scattering from $P\left(x_{p}, y_{p}, 0\right)$ can be written as

$$
\begin{aligned}
s\left(\widehat{t}, t_{m}\right)= & w_{r}\left(\hat{t}-\frac{R_{\mathrm{bf}}\left(t_{m}\right)}{c}\right) w_{a}\left(t_{m}\right) \\
& \times \exp \left[j \pi \gamma\left(\widehat{t}-\frac{R_{\mathrm{bf}}\left(t_{m}\right)}{c}\right)^{2}\right] \\
& \times \exp \left[-j \frac{2 \pi}{\lambda} R_{\mathrm{bf}}\left(t_{m}\right)\right],
\end{aligned}
$$

where $\widehat{t}$ is the range time, $\lambda$ is the wavelength, $c$ is the speed of light, and $\gamma$ is the chirp rate. $w_{r}(\cdot)$ and $w_{a}(\cdot)$ are the range and azimuth envelopes, respectively.

To design imaging algorithms for MBFL-SAR more conveniently, it is important to take efficient approximation of $R_{\mathrm{bf}}\left(t_{m}\right)$; some simulations are given using the parameters listed in Table 1 and the results are shown in Figure 2.

It can be seen that the maximum approximation error when keeping the terms up to the quadratic term is about $0.02 \mathrm{~m}$, nearly the value of wavelength, so it is necessary to keep the higher term. When keeping the terms up to the third term, the maximum approximation error is only 2.44 $\times 10^{-6} \mathrm{~m}$, which is much less than the value of wavelength. Therefore, expanding (3) as a Taylor series of $t_{m}$ needs to keep the terms up to the third-order

$$
R_{\mathrm{bf}}\left(t_{m}\right)=R_{\mathrm{bf0}}+k_{1} t_{m}+k_{2} t_{m}^{2}+k_{3} t_{m}^{3}+o\left(t_{m}^{3}\right),
$$

with

$$
\begin{gathered}
R_{\mathrm{bf} 0}=R_{r \mathrm{cen}}+R_{t \mathrm{cen}}, \\
k_{1}=\frac{\mu_{r 1}}{2 R_{r \mathrm{cen}}}+\frac{\mu_{t 1}}{2 R_{t \mathrm{cen}}}, \\
k_{2}=\frac{\mu_{r 2}}{2 R_{r \mathrm{cen}}}-\frac{\mu_{r 1}^{2}}{8 R_{r \mathrm{cen}}^{3}}+\frac{\mu_{t 2}}{2 R_{t \mathrm{cen}}}-\frac{\mu_{t 1}^{2}}{8 R_{t \mathrm{cen}}^{3}}, \\
k_{3}=\frac{\mu_{r 3}}{2 R_{r \mathrm{cen}}}-\frac{\mu_{r 1} \mu_{r 2}}{4 R_{r \mathrm{cen}}^{3}}+\frac{\mu_{r 1}^{3}}{16 R_{r \mathrm{cen}}^{5}} \\
+\frac{\mu_{t 3}}{2 R_{t \mathrm{cen}}}-\frac{\mu_{t 1} \mu_{t 2}}{4 R_{t \mathrm{cen}}^{3}}+\frac{\mu_{t 1}^{3}}{16 R_{t \mathrm{cen}}^{5}} .
\end{gathered}
$$

2.2.1. Doppler Frequency and Doppler Centroid. Doppler frequency can be obtained through derivation of slant range and is expressed as

$$
f\left(t_{m}\right)=-\frac{1}{\lambda} \frac{d R_{\mathrm{bf}}\left(t_{m}\right)}{d t_{m}}=-\frac{1}{\lambda}\left(k_{1}+2 k_{2} t_{m}+3 k_{3} t_{m}^{2}\right) .
$$

TABLE 1: Parameters used in the simulations.

\begin{tabular}{lc}
\hline Wavelength & $0.02 \mathrm{~m}$ \\
Bandwidth & $50 \mathrm{MHz}$ \\
Sampling frequency & $100 \mathrm{MHz}$ \\
Pulse duration & $2 \mu \mathrm{s}$ \\
PRF & $9 \mathrm{KHz}$ \\
$\psi$ & $20^{\circ}$ \\
$H_{T}$ & $25 \mathrm{~km}$ \\
$H_{R}$ & $20 \mathrm{~km}$ \\
$\vec{v}_{t 0}$ & $(0,1500,-1000) \mathrm{m} / \mathrm{s}$ \\
$\vec{a}_{t}$ & $(0,-50,30) \mathrm{m} / \mathrm{s}^{2}$ \\
$\vec{v}_{r 0}$ & $(0,1500,-1000) \mathrm{m} / \mathrm{s}$ \\
$\vec{a}_{r}$ & $(0,-50,30) \mathrm{m} / \mathrm{s}^{2}$ \\
\hline
\end{tabular}

Doppler centroid represents echo Doppler frequency at the moment beam center cross target, given by

$$
f_{\mathrm{dc}}=-\left.\frac{1}{\lambda} \frac{d R_{\mathrm{bf}}\left(t_{m}\right)}{d t_{m}}\right|_{t_{m}=0}=-\frac{1}{\lambda} k_{1}=-\frac{1}{\lambda}\left(\frac{\mu_{r 1}}{2 R_{r \mathrm{cen}}}+\frac{\mu_{t 1}}{2 R_{\mathrm{tcen}}}\right) .
$$

Doppler centroid represents correlativity only to motion parameters and irrelevance to location of targets in the traditional case where monostatic SAR moves with invariant velocity along a straight line or the case where transmitter and receiver move with equal velocities along parallel trajectories in traditional airborne bistatic SAR system. However, it can be seen from (9) that Doppler centroid in MBFL-SAR conducts different performance that $f_{\mathrm{dc}}$ varies with such parameters correlative to location of targets as $R_{r c e n}, R_{t c e n}, \mu_{r 1}$, and $\mu_{t 1}$, so $f_{\mathrm{dc}}$ is space-variant in MBFL-SAR configuration.

2.2.2. Doppler Frequency Rate. Doppler frequency rate is the change rate of Doppler frequency and can be expressed as

$$
\begin{aligned}
\gamma_{a} & =-\left.\frac{1}{\lambda} \frac{d^{2} R_{\mathrm{bf}}\left(t_{m}\right)}{d t_{m}^{2}}\right|_{t_{m}=0} \\
& =-\frac{1}{\lambda}\left[\frac{\mu_{r 2}}{R_{r \mathrm{cen}}}-\frac{\mu_{r 1}^{2}}{4 R_{r \mathrm{cen}}^{3}}+\frac{\mu_{t 2}}{R_{t \mathrm{cen}}}-\frac{\mu_{t 1}^{2}}{4 R_{\mathrm{tcen}}^{3}}\right] .
\end{aligned}
$$

We can find from (2.1) and (10) that Doppler frequency rate not only depends on the coordinates of targets but also relates strongly to original velocities and accelerations of platforms in this special configuration. Given such consumption that transmitter and receiver have equivalent velocities and invariant moving heights and accelerations are ignored and that the included angle $\psi$ is consumed as $0^{\circ}$, we can get the traditional bistatic parallel and velocity-equivalent mode. Therefore, the traditional bistatic parallel and velocityequivalent mode is the special case of MBFL-SAR to some extent. 


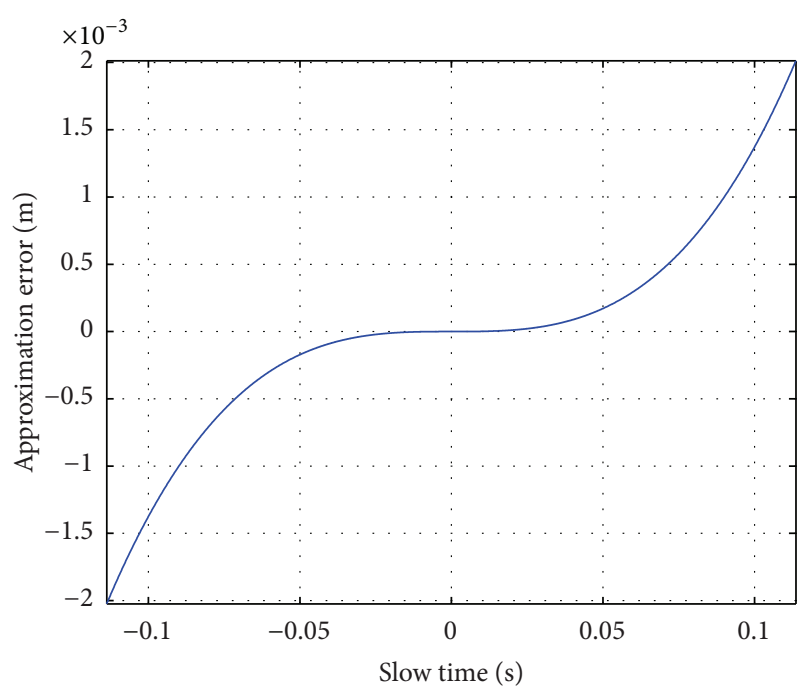

(a) Up to quadratic term

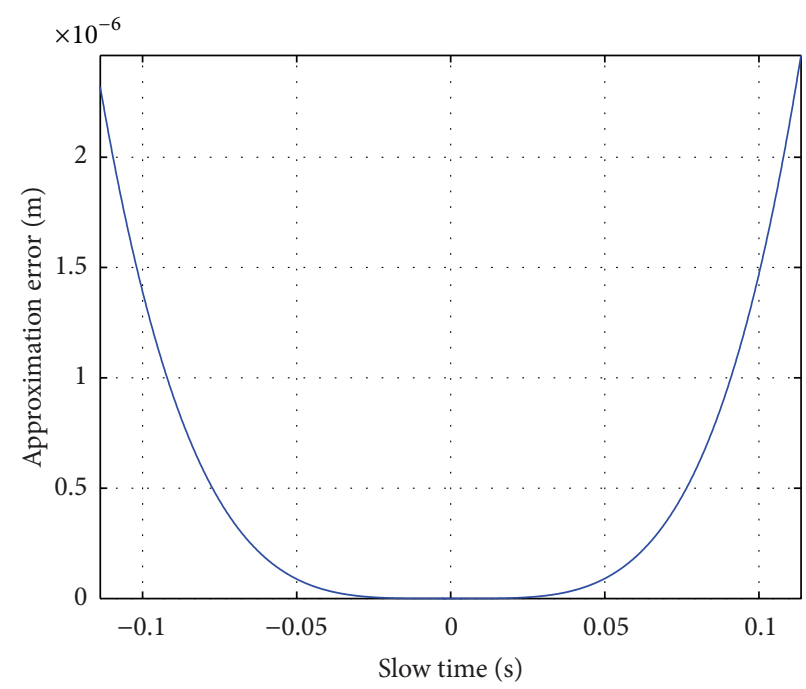

(b) Up to the third term

FIgURE 2: Approximation errors of the bistatic slant range.

2.2.3. Range Walk Ratio ( $R W R$ ). Range walk ratio represents the range walk increment of echo signal in per time unit; it can be obtained as

$$
\mathrm{RWR}=\frac{d\left(k_{1} t_{m}\right)}{d t_{m}}=k_{1}=\frac{\mu_{r 1}}{2 R_{r \text { cen }}}+\frac{\mu_{t 1}}{2 R_{t c e n}} .
$$

Similar to Doppler centroid, range walk ratio in MBFLSAR configuration is also space-variant. Such space-variance leads to the result that MBFL-SAR does not have azimuthinvariant property, which is important to conventional algorithms in monostatic SAR and airborne bistatic SAR in parallel and velocity-equivalent mode. So space-variance must be considered when the range cell migration correction is achieved.

\section{Analysis of 2D Resolution}

In this section, 2D imagingresolution of MBFL-SAR is analyzed. The expressions of range resolution and Doppler resolution are determined using gradient method [24]. Then key parameters affecting the $2 \mathrm{D}$ imaging ability are found out, which is helpful to design system parameters and motion parameters.

3.1. Range Resolution. Assume that $\mathbf{R}_{T}$ and $\mathbf{R}_{R}$ represent slant range vectors from transmitter and receiver to target at $t_{m}$, respectively, and can be written as

$$
\begin{aligned}
& \mathbf{R}_{T}=\left(x_{t}-x_{p}\right) \mathbf{i}+\left(y_{t}-y_{p}\right) \mathbf{j}+z_{t} \mathbf{k}, \\
& \mathbf{R}_{R}=\left(x_{r}-x_{p}\right) \mathbf{i}+\left(y_{r}-y_{p}\right) \mathbf{j}+z_{r} \mathbf{k},
\end{aligned}
$$

where $\mathbf{i}, \mathbf{j}$, and $\mathbf{k}$ are unit vectors in the directions $x$ axis, $y$ axis, and $z$ axis.
According to the geometrical relationship in Figure 1, we can determine the coefficients in (12) such that

$$
\begin{gathered}
x_{t}-x_{p}=R_{T}\left(\cos \theta_{T} \sin \phi_{T} \cos \psi-\sin \theta_{T} \sin \psi\right), \\
x_{r}-x_{p}=-R_{R} \sin \theta_{R}, \\
y_{t}-y_{p}=-R_{T}\left(\sin \theta_{T} \cos \psi+\cos \theta_{T} \sin \phi_{T} \sin \psi\right), \\
y_{r}-y_{p}=-R_{R} \cos \theta_{R} \sin \phi_{R},
\end{gathered}
$$

where $\theta_{T}$ and $\phi_{T}$ are squint angle and look angle of transmitter, respectively, $\theta_{R}$ and $\phi_{R}$ are angle deviating from the beam center and look angle of receiver, respectively, and the angles are as follows:

$\theta_{T}$

$$
\begin{gathered}
=\arcsin \left(\frac{\left|\left(y_{p}-y_{t}\right)+\left(x_{p}-x_{t}\right) \tan \psi\right|}{\sqrt{\left(y_{p}-y_{t}\right)^{2}+\left(x_{p}-x_{t}\right)^{2}+z_{t}^{2}} \sqrt{\tan ^{2} \psi+1}}\right), \\
\phi_{T}=\arcsin \left(\frac{z_{t}}{\cos \theta_{T} \sqrt{\left(y_{p}-y_{t}\right)^{2}+\left(x_{p}-x_{t}\right)^{2}+z_{t}^{2}}}\right), \\
\theta_{R}=\arcsin \left(\frac{x_{p}-x_{r}}{\sqrt{\left(y_{p}-y_{r}\right)^{2}+\left(x_{p}-x_{r}\right)^{2}+z_{r}^{2}}}\right), \\
\phi_{R}=\arcsin \left(\frac{y_{p}-y_{r}}{\cos \theta_{R} \sqrt{\left(y_{p}-y_{r}\right)^{2}+\left(x_{p}-x_{r}\right)^{2}+z_{r}^{2}}}\right) .
\end{gathered}
$$


Substituting (13) into (12) and rearranging the result, we can obtain the unit vectors $\mathbf{i}_{R_{T}}$ and $\mathbf{i}_{R_{R}}$ from transmitter and receiver to $P\left(x_{p}, y_{p}, 0\right)$, respectively, expressed as

$$
\begin{aligned}
\mathbf{i}_{R_{T}}= & \left(\cos \theta_{T} \sin \phi_{T} \cos \psi-\sin \theta_{T} \sin \psi\right) \mathbf{i} \\
& -\left(\sin \theta_{T} \cos \psi+\cos \theta_{T} \sin \phi_{T} \sin \psi\right) \mathbf{j}+\frac{z_{t}}{R_{T}} \mathbf{k} \\
& \mathbf{i}_{R_{R}}=-\sin \theta_{R} \mathbf{i}-\cos \theta_{R} \sin \phi_{R} \mathbf{j}+\frac{z_{r}}{R_{R}} \mathbf{k} .
\end{aligned}
$$

According to gradient method in [24], we can get the range resolution of MBFL-SAR:

$$
\begin{gathered}
\rho_{g}=\frac{c}{B} \times\left(\left(\cos \theta_{T} \sin \phi_{T} \cos \psi-\sin \theta_{T} \sin \psi-\sin \theta_{R}\right)^{2}\right. \\
+\left[\sin \theta_{T} \cos \psi+\cos \theta_{T} \sin \phi_{T} \sin \psi\right. \\
\left.\left.+\cos \theta_{R} \sin \phi_{R}\right]^{2}\right)^{-1 / 2}
\end{gathered}
$$

where $B$ is bandwidth of transmitted signal and $\rho_{g}$ is the range resolution which reflects the capability of distinguishing two targets in horizontal plane of imaging area. Given a certain bandwidth $B, \rho_{g}$ is related to such angles between radars and target as $\theta_{T}, \phi_{T}, \theta_{R}$, and $\phi_{R}$ and included angle $\psi$ (collectively called "space angles"). Although heights of transmitter and receiver do not appear in (16) explicitly, they can also affect $\rho_{g}$ through such "space angles." In addition, the values of $\rho_{g}$ are different because of different space angles caused by different coordinates of targets and different slow time $t_{m}$. Thus, $\rho_{g}$ is not only space-variant but also time-variant.

3.2. Doppler Resolution. As shown in the coordinate system in Figure 1, velocity vectors of transmitter and receiver platforms are expressed as

$$
\begin{gathered}
\mathbf{V}_{T}=V_{T g} \sin \psi \mathbf{i}+V_{T g} \cos \psi \mathbf{j}-V_{T z} \mathbf{k}, \\
\mathbf{V}_{R}=0 \mathbf{i}+V_{R g} \mathbf{j}-V_{R z} \mathbf{k},
\end{gathered}
$$

where $V_{T g}$ and $V_{R g}$ represent speed components of transmitter and receiver in horizontal plane, respectively, and $V_{T z}$ and $V_{R z}$ denote speed components of transmitter and receiver in the vertical plane. Because the directions of motion of transmitter and receiver in the vertical plane are downward, speed components in vertical direction are negative.

Again, using the gradient method, the Doppler resolution can be obtained as

$$
\begin{aligned}
\rho_{a}=\frac{\lambda}{T_{a}} \times( & {\left[\omega_{T g}\left(\cos ^{2} \theta_{T} \sin \psi+\Theta_{1} \cos \psi\right)\right.} \\
& +\omega_{T z}\left(\Theta_{2} \cos \psi-\sin \theta_{T} \sin \psi\right) \\
& \left.-\omega_{R g} \Theta_{3}-\omega_{R z} \sin \theta_{R}\right]^{2} \\
+ & {\left[\omega_{T g}\left(\cos ^{2} \theta_{T} \cos \psi-\Theta_{1} \sin \psi\right)\right.} \\
& -\omega_{T z}\left(\sin \theta_{T} \cos \psi+\Theta_{2} \sin \psi\right) \\
& \left.\left.+\omega_{R g} \Theta_{4}-\omega_{R z} \sin \phi_{R} \cos \theta_{R}\right]^{2}\right)^{-1 / 2}
\end{aligned}
$$

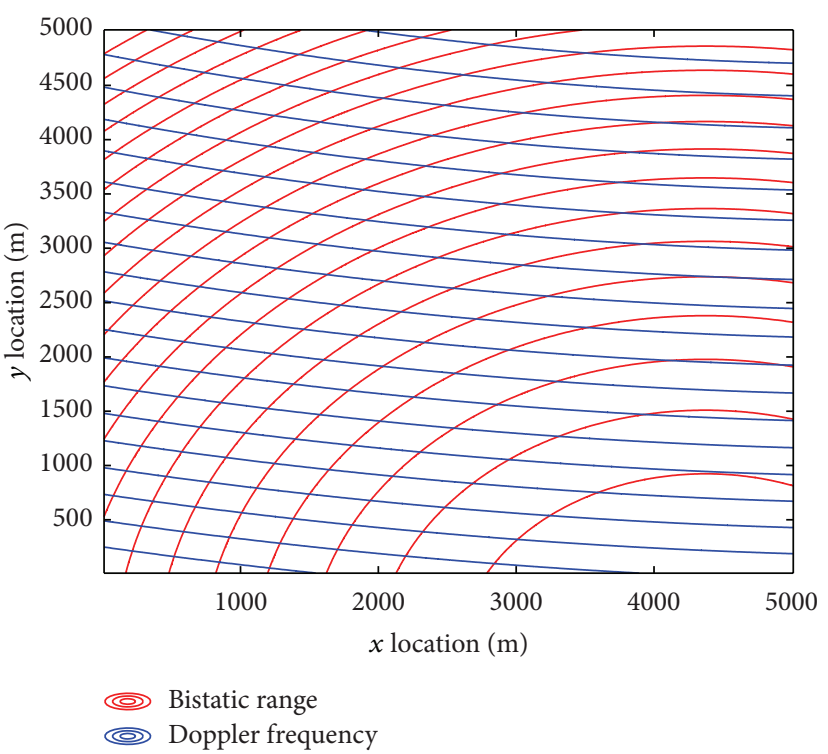

FIGURE 3: MBFL-SAR range contours and Doppler frequency contours when $t_{m}=0$.

with

$$
\begin{gathered}
\omega_{T g}=\frac{V_{T g}}{R_{T}}, \quad \Theta_{1}=\sin \theta_{T} \sin \phi_{T} \cos \theta_{T}, \\
\omega_{T z}=\frac{V_{T z}}{R_{T}} \frac{z_{T}}{R_{T}}, \quad \Theta_{2}=\sin \phi_{T} \cos \theta_{T}, \\
\omega_{R g}=\frac{V_{R g}}{R_{R}}, \quad \Theta_{3}=\sin \phi_{R} \sin \theta_{R} \cos \theta_{R}, \\
\omega_{R z}=\frac{V_{R z}}{R_{R}} \frac{z_{R}}{R_{R}}, \quad \Theta_{4}=1-\sin ^{2} \phi_{R} \cos ^{2} \theta_{R} .
\end{gathered}
$$

Similarly, $\rho_{a}$ is also dependent on "space angles." Besides, synthetic aperture time $T_{a}$ and signal wavelength $\lambda$ can also have influence on $\rho_{a}$. Similar to $\rho_{g}, \rho_{a}$ is space-variant and time-variant too.

\section{Numerical Results}

In this section, several examples are provided to illustrate the resolution ability in the MBFL-SAR. Below are some simulation results of the resolution capability and 2D resolution. Parameters used in the simulations are listed in Table 1. Due to the features of space-variance and time-variance, we give the results at a certain time (here, $t_{m}=0$ ). Figure 3 depicts MBFL-SAR range contours and Doppler frequency contours, and MBFL-SAR range resolution and Doppler resolution are given in Figure 4. To test the time-variant property, some simulations of the $2 \mathrm{D}$ resolution of the point target located at $(0,4500,0)$ at different slow time are also implemented, and the results are shown in Figure 5.

To further analyze the signal properties of MBFL-SAR, several simulations about airborne bistatic forward-looking SAR (ABFL-SAR) are also realized, where the velocities of the transmitter and receiver are $150 \mathrm{~m} / \mathrm{s}$ and $100 \mathrm{~m} / \mathrm{s}$ with 


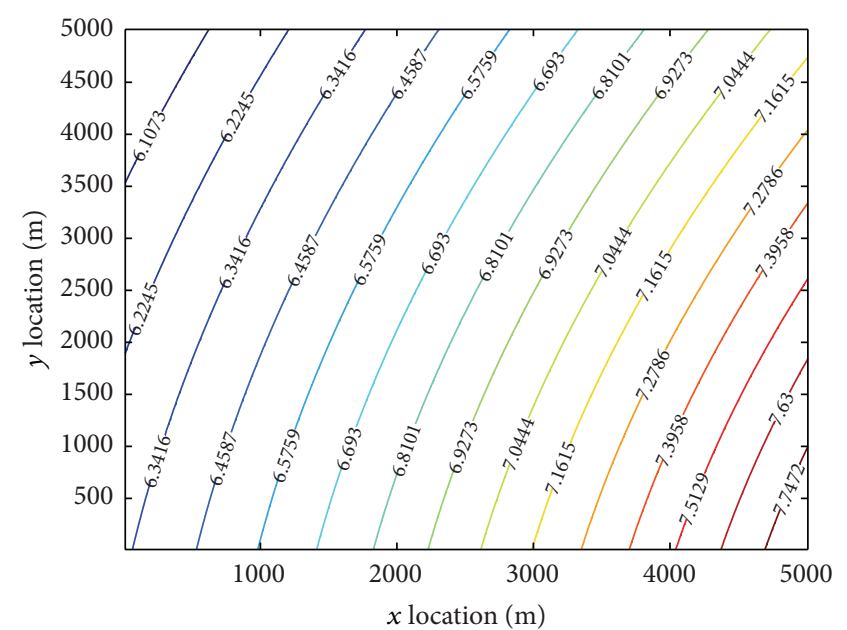

(a) Range resolution

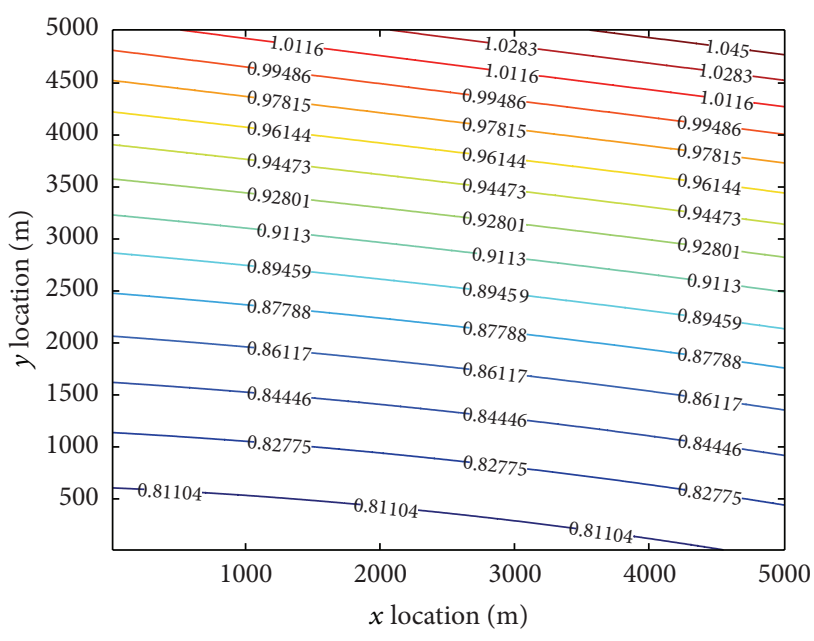

(b) Doppler resolution

FIGURE 4: MBFL-SAR range resolution and Doppler resolution when $t_{m}=0$.

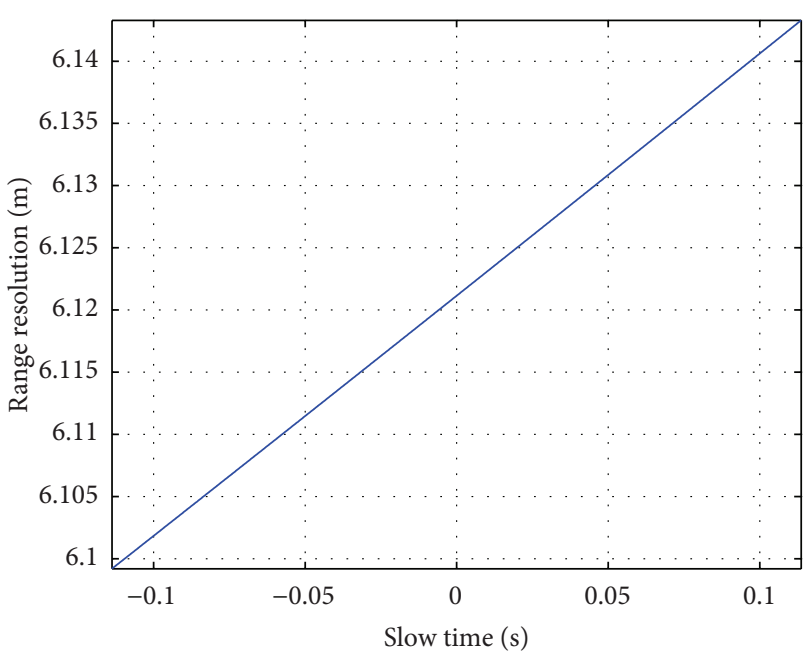

(a) Range resolution

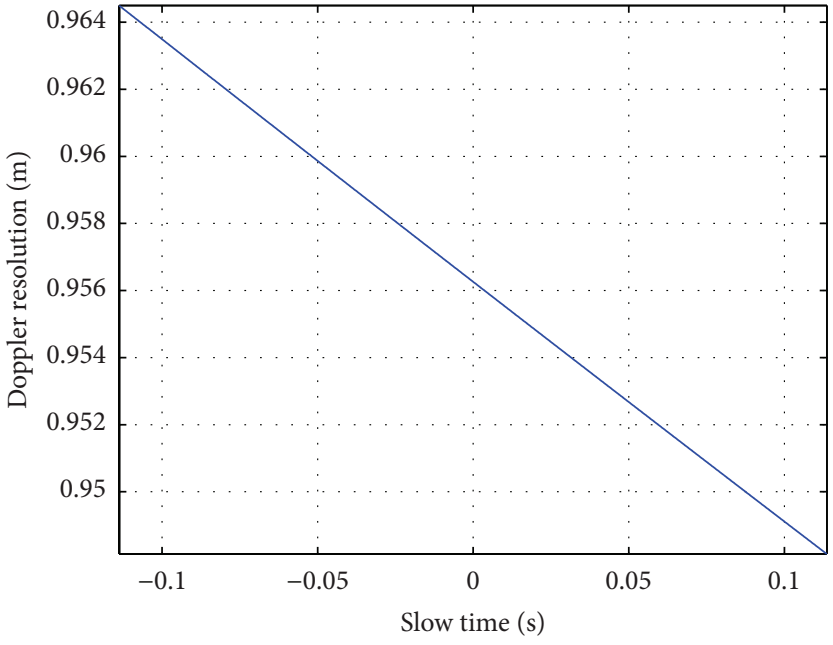

(b) Doppler resolution

FIgURE 5: MBFL-SAR range resolution and Doppler resolution at different $t_{m}$.

included angle of 20 degrees, and the heights are $5 \mathrm{~km}$ and $2 \mathrm{~km}$, respectively. Doppler frequency and RWR comparisons of five-point targets $150 \mathrm{~m}$ apart from each other in the imaging area are conducted between ABFL-SAR and MBFLSAR during the same synthetic aperture time. The results are given in Figures 6 and 7, respectively. Besides, experiments on range cell curvature (RCC) of one-point target are finished and results are in Figure 8.

MBFL-SAR range contours and Doppler frequency contours when $t_{m}=0$ are shown in Figure 3, where range contours and Doppler frequency contours are intersectant but all of them are not perpendicular. It can be seen that MBFL-SAR configuration has the capability of $2 \mathrm{D}$ imaging resolution. In addition, range contours and Doppler frequency contours are nearly perpendicular in some places of the imaging area but are nearly parallel in some other places, which describe the space-variant feature of $2 \mathrm{D}$ resolution as discussed previously.
Figure 4 represents the results of MBFL-SAR range resolution and Doppler resolution when $t_{m}=0$. Range resolution is shown in Figure 4(a), where we can find that the values of range resolution vary with the location of the targets in the imaging area. And because of the particularity of MBFL-SAR configuration, there exist isolines among different targets located in different $x$ location and $y$ location; for example, two targets located in $(1350,1510)$ and $(2220,3810)$ have equal value of $6.5759 \mathrm{~m}$. Unlike range resolution, the value of Doppler resolution is very low, which can be seen from Figure 4(b). The space-variant property of Doppler resolution can also be found from the same value of $0.9113 \mathrm{~m}$ of two targets located in $(810,3130)$ and $(3640,2720)$, respectively. In addition, targets located in smaller $x / y$ location conduct better performance than ones located in greater $x / y$ location in MBFL-SAR configuration. 


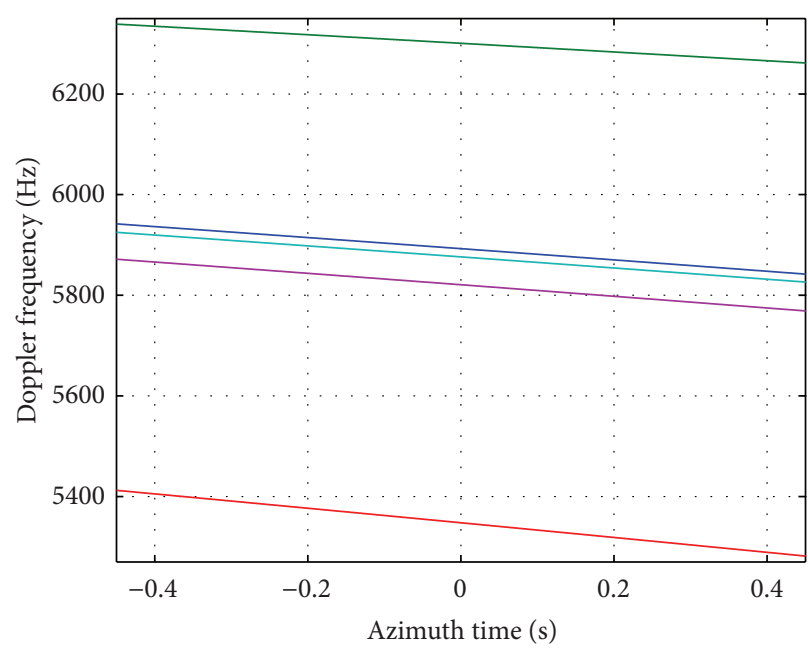

(a) ABFL-SAR

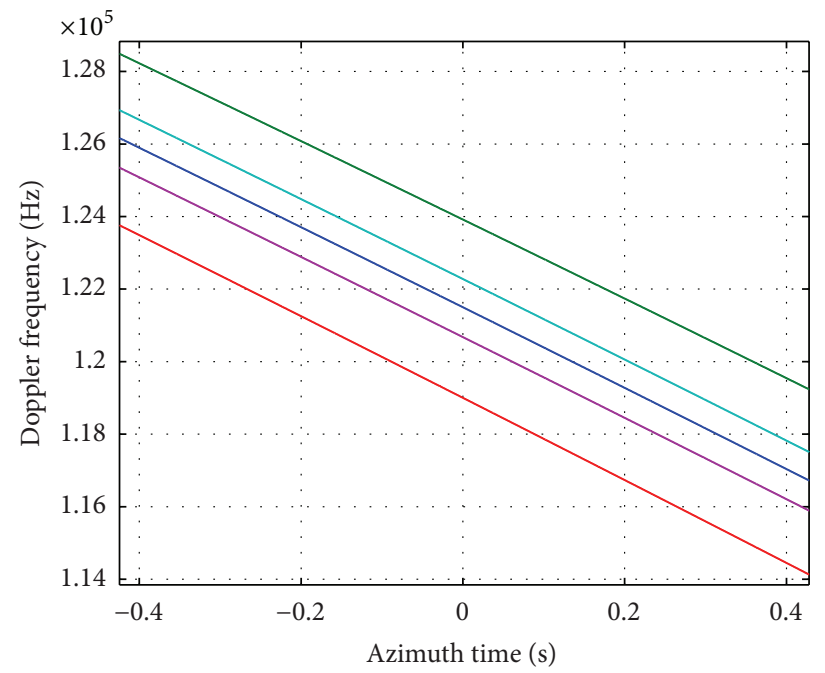

(b) MBFL-SAR

Figure 6: Doppler frequency comparison between ABFL-SAR and MBFL-SAR.

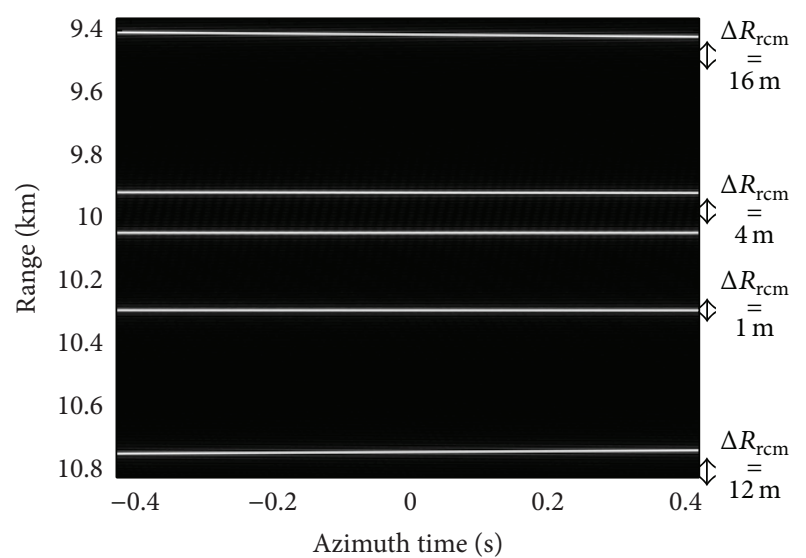

(a) ABFL-SAR

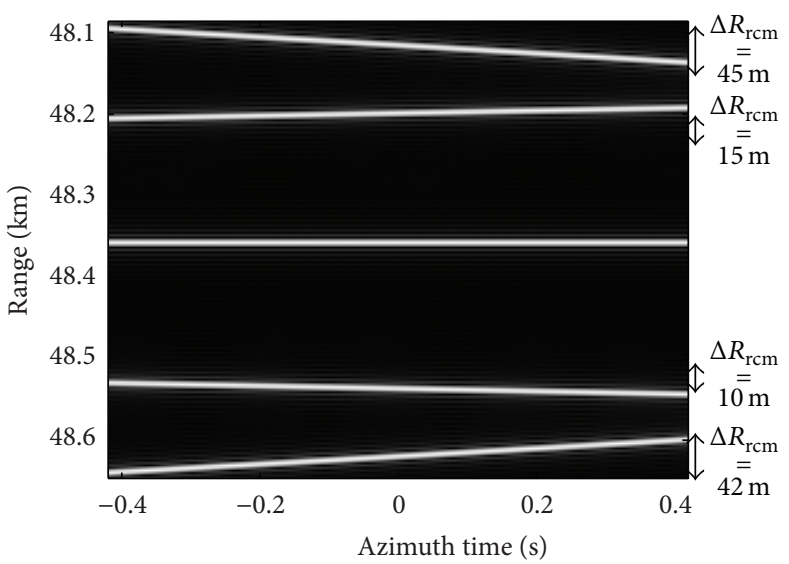

(b) MBFL-SAR

FIGURE 7: RWR comparison between ABFL-SAR and MBFL-SAR.

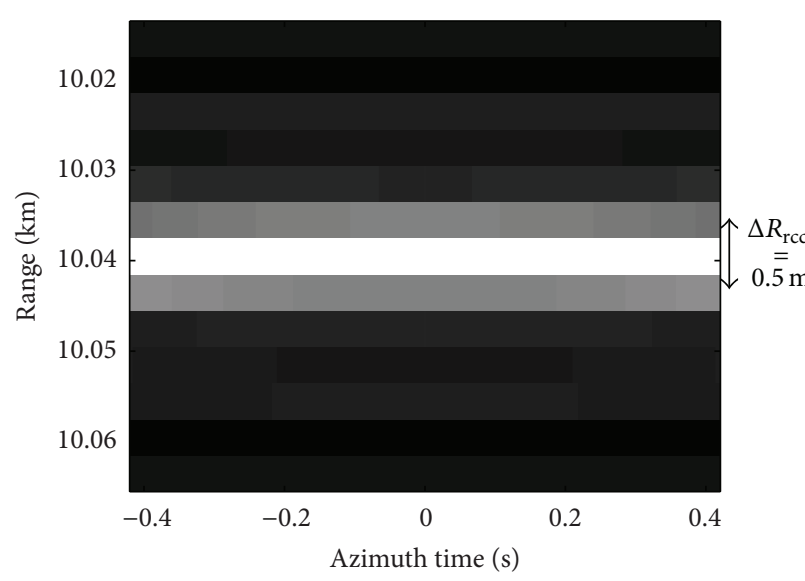

(a) ABFL-SAR

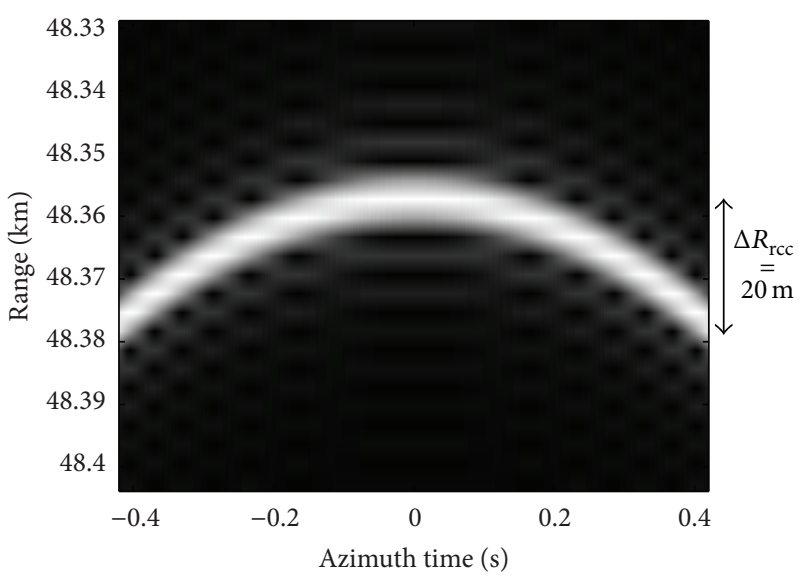

(b) MBFL-SAR

Figure 8: RCC comparison between ABFL-SAR and MBFL-SAR. 
International Journal of Antennas and Propagation

Some simulations are also achieved to analyze range resolution and Doppler resolution of MBFL-SAR at different $t_{m}$, and the results are like Figure 5. Range resolution and Doppler resolution vary with $t_{m}$ but the changes are tiny during the synthetic aperture time, $0.044 \mathrm{~m}$ of range resolution and $0.016 \mathrm{~m}$ of Doppler resolution, respectively. This characteristic can be considered when designing the imaging algorithms of this configuration, and some approximations could be taken.

Because of the presence of high velocities and accelerations in MBFL-SAR configuration, Doppler frequencies of targets in the imaging area have greater changes than ABFLSAR. And range cell migrations (RCMs) are also more severe during the same synthetic aperture time; that is to say, RWRs are greater. As shown in Figure 6, Doppler frequency curves in both configurations are nearly parallel linear lines, but the maximum changes of Doppler frequency in ABFL-SAR are only $131 \mathrm{~Hz}$, while the one in MBFL-SAR is much greater, about $9600 \mathrm{~Hz}$. Results given in Figure 7 show that RWRs in both configurations are space-variant, but the one in MBFLSAR is more grievous. The maximum RCM in ABFL-SAR is about $16 \mathrm{~m}$, while the one during the same synthetic aperture time in MBFL-SAR is $45 \mathrm{~m}$, which results from the fact that the range in MBFL-SAR is much further and changes more rapidly than ABFL-SAR. Figure 8 depicts RCC comparison between ABFL-SAR and MBFL-SAR, where we can find that RCC in MBFL-SAR is more serious than ABFL-SAR, about $20 \mathrm{~m}$ and $2 \mathrm{~m}$, respectively. The greater RCC could not be neglected in MBFL-SAR when designing imaging algorithms, while the one in ABFL-SAR can be ignored if the influence on the resolution is small enough.

\section{Conclusions}

Applications of MIMO radar networking technology to radar imaging can improve the performance of radar imaging resolution, targets detection, recognition, and tracking. As a special MIMO radar networking system, MBFL-SAR is a special mode of bistatic forward-looking imaging by combining geometry configuration of bistatic motion with forwardlooking mode and has potential of being applied to missile precision terminal guidance. Properties of MBFL-SAR are analyzed in this paper. The high velocities and the two squareroot terms lead to the presence of high-order terms in slant range history and Doppler history which cannot be ignored. Simulations have been done to illustrate the resolution ability of this configuration, and both range resolution and Doppler resolution are space-variant and time-variant. The tiny changes of the values of $2 \mathrm{D}$ resolution in this configuration during short synthetic aperture time and comparisons with ABFL-SAR can be used to design imaging algorithms in this special configuration. Research on the properties of MBFL-SAR configuration validates the efficiency of the application of MIMO radar networking to radar imaging, which also provides theoretical groundwork for the whole system parameter design and imaging algorithms of MBFLSAR.

\section{Conflict of Interests}

The authors declare that there is no conflict of interests regarding the publication of this paper.

\section{Acknowledgments}

This work was supported by the National Natural Science Foundation of China under Grant nos. 61001211 and 61303035, the Fundamental Research Funds for the Central Universities (K5051202016), and the Science Foundation for Navigation (20110181004).

\section{References}

[1] E. Fishler, A. Haimovich, R. Blum, D. Chizhik, L. Cimini, and R. Valenzuela, "MIMO radar: an idea whose time has come," in Proceedings of the IEEE Radar Conference, pp. 71-78, April 2004.

[2] X. Li, Z. Zhang, W. Mao, X. Wang, J. Lu, and W. Wang, "A study of frequency diversity MIMO radar beamforming," in Proceedings of the IEEE 10th International Conference on Signal Processing (ICSP '10), pp. 352-356, October 2010.

[3] R. Sharma, "Analysis of MIMO radar ambiguity functions and implications on clear region," in Proceedings of the IEEE International Radar Conference (RADAR '10), pp. 544-548, June 2010.

[4] J. Li and P. Stoica, MIMO Radar Signal Processing, John Wiley \& Sons, Hoboken, NJ, USA, 2009.

[5] I. Cumming and F. H. Wong, Digital Processing of Synthetic Aperture Radar Data: Algorithms and Implementation, Artech House, Norwood, Mass, USA, 2004.

[6] J. Li, G. Liao, and H. Griffiths, "Bistatic MIMO radar space-time adaptive processing," in Proceedings of the IEEE Radar Conference: In the Eye of the Storm (RadarCon '11), pp. 498-501, May 2011.

[7] X. H. Wu, A. A. Kishk, and A. W. Glisson, "MIMO-OFDM radar for direction estimation," IET Radar, Sonar \& Navigation, vol. 4, no. 1, pp. 28-36, 2010.

[8] J. Li and P. Stoica, "MIMO radar with colocated antennas," IEEE Signal Processing Magazine, vol. 24, no. 5, pp. 106-114, 2007.

[9] A. Haimovich, R. Blum, and L. Cimini, "MIMO radar with widely separated antennas," IEEE Signal Processing Magazine, vol. 25, no. 1, pp. 116-129, 2008.

[10] Z. Shi, H. Wang, P. Zhang, X. Tang, B. Wu, and C. Wang, "Study on the probability of successful handoff of missile trajectory from midcourse guidance to terminal guidance," in Proceedings of the International Conference on Computational and Information Sciences (ICCIS '10), pp. 1005-1008, Chengdu, China, December 2010.

[11] Q. Zhao, X. Zhang, W. Song et al., "Rotational analysis of certain terminal-guidance projectile," in Proceedings of the International Conference on Electronic Measurement and Instruments (ICEMI '07), pp. 415-418, July 2007.

[12] F. Comblet, A. Khenchaf, A. Baussard et al., "Bistatic synthetic aperture radar imaging: theory, simulations, and validations," IEEE Transactions on Antennas and Propagation, vol. 54, no. 11, pp. 3529-3540, 2006.

[13] I. Walterscheid, T. Espeter, J. Klare, A. R. Brenner, and J. H. G. Ender, "Potential and limitations of forward-looking bistatic SAR," in Proceedings of the IEEE International Geoscience and Remote Sensing Symposium (IGARSS '10), pp. 216-219, Honolulu, Hawaii, USA, July 2010. 
[14] W. Wang, "Approach of adaptive synchronization for bistatic SAR real-time imaging," IEEE Transactions on Geoscience and Remote Sensing, vol. 45, no. 9, pp. 2695-2700, 2007.

[15] W. Wang, C. Ding, and X. Liang, "Time and phase synchronisation via direct-path signal for bistatic synthetic aperture radar systems," IET Radar, Sonar and Navigation, vol. 2, no. 1, pp. 1-11, 2008.

[16] M. Weib, "Synchronisation of bistatic radar systems," in Proceedings of IEEE International Geoscience and Remote Sensing Symposium (IGARSS '04), pp. 1750-1753, September 2004.

[17] J. Wu, J. Yang, H. Yang, and Y. Huang, "Optimal geometry configuration of bistatic forward-looking SAR," in Proceedings of the IEEE International Conference on Acoustics, Speech and Signal Processing (ICASSP '09), pp. 1117-1120, 2009.

[18] X. Qiu and D. Hu, "Some reflections on bistatic SAR of forwardlooking configuration," IEEE Geoscience and Remote Sensing Letters, vol. 5, no. 4, pp. 735-739, 2008.

[19] T. Espeter, I. Walterscheid, J. Klare et al., "Bistatic forwardlooking SAR: results of a spaceborne-airborne experiment," IEEE Geoscience and Remote Sensing Letters, vol. 8, no. 4, pp. 765-768, 2011.

[20] J. Balke, "Field test of bistatic forward-looking synthetic aperture radar," in Proceedings of IEEE International Radar Conference, pp. 424-429, May 2005.

[21] Z. Li, D. Yao, and T. Long, "SPECAN algorithm for forwardlooking bistatic SAR," in Proceedings of 9th International Conference on Signal Processing ( ICSP '08), pp. 2517-2520, October 2008.

[22] R. Wang, O. Loffeld, H. Nies, and V. Peters, "Image formation algorithm for bistatic forward-looking SAR," in Proceedings of the 30th IEEE International Geoscience and Remote Sensing Symposium (IGARSS '10), pp. 4091-4094, Honolulu, Hawaii, USA, July 2010.

[23] J. Wu, Z. Li, and Y. Huang, "Focusing bistatic forward-looking SAR with stationary transmitter based on keystone transform and nonlinear chirp scaling," IEEE Geoscience and Remote Sensing Letters, vol. 11, no. 1, pp. 148-152, 2014.

[24] G. P. Cardillo, "On the use of gradient to determine bistatic SAR resolution," in Proceedings of Antennas and Propagation Society International Symposium, vol. 2, pp. 1032-1035, May 1990. 

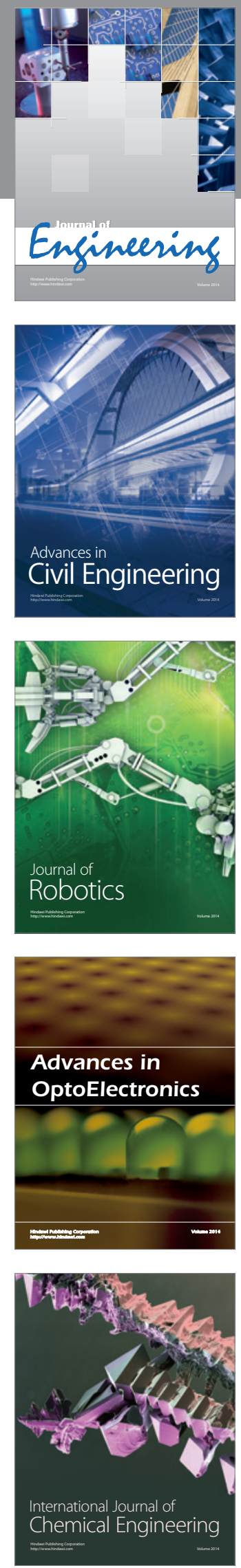

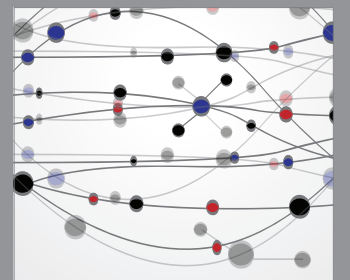

The Scientific World Journal
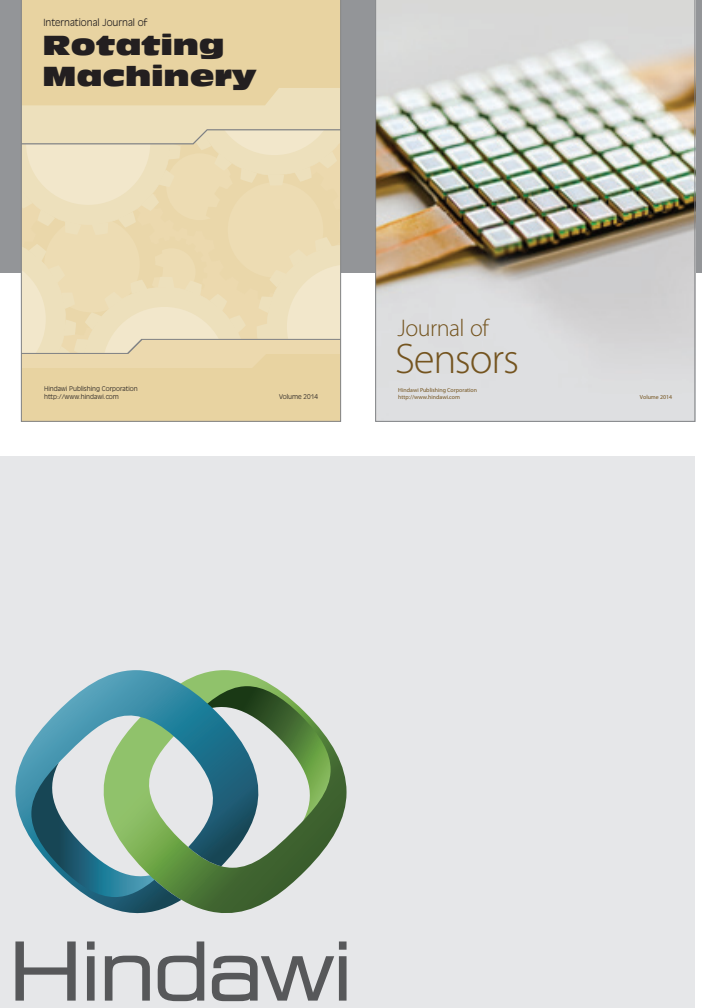

Submit your manuscripts at http://www.hindawi.com
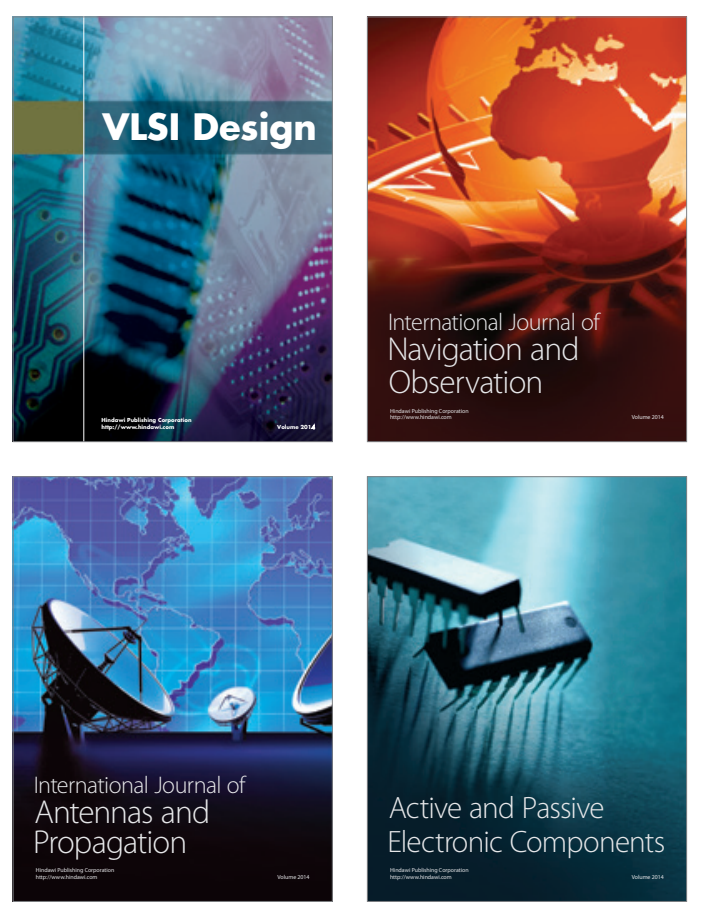
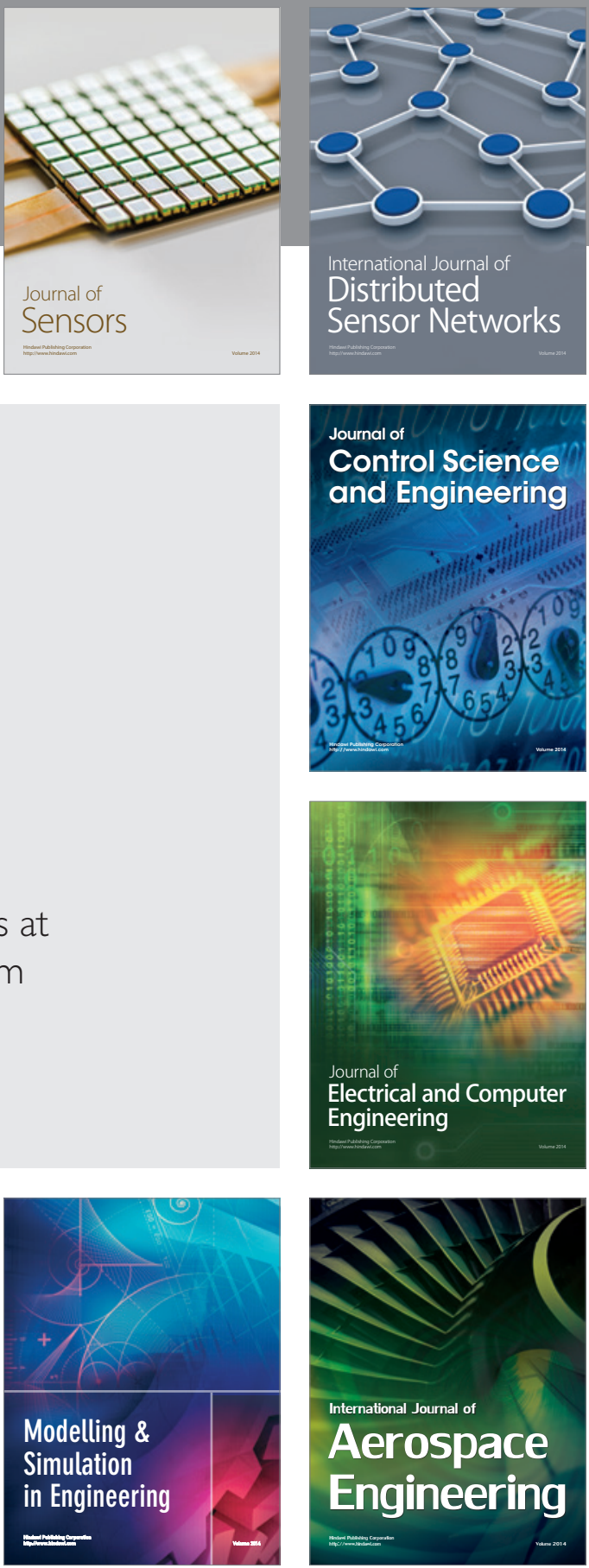

Journal of

Control Science

and Engineering
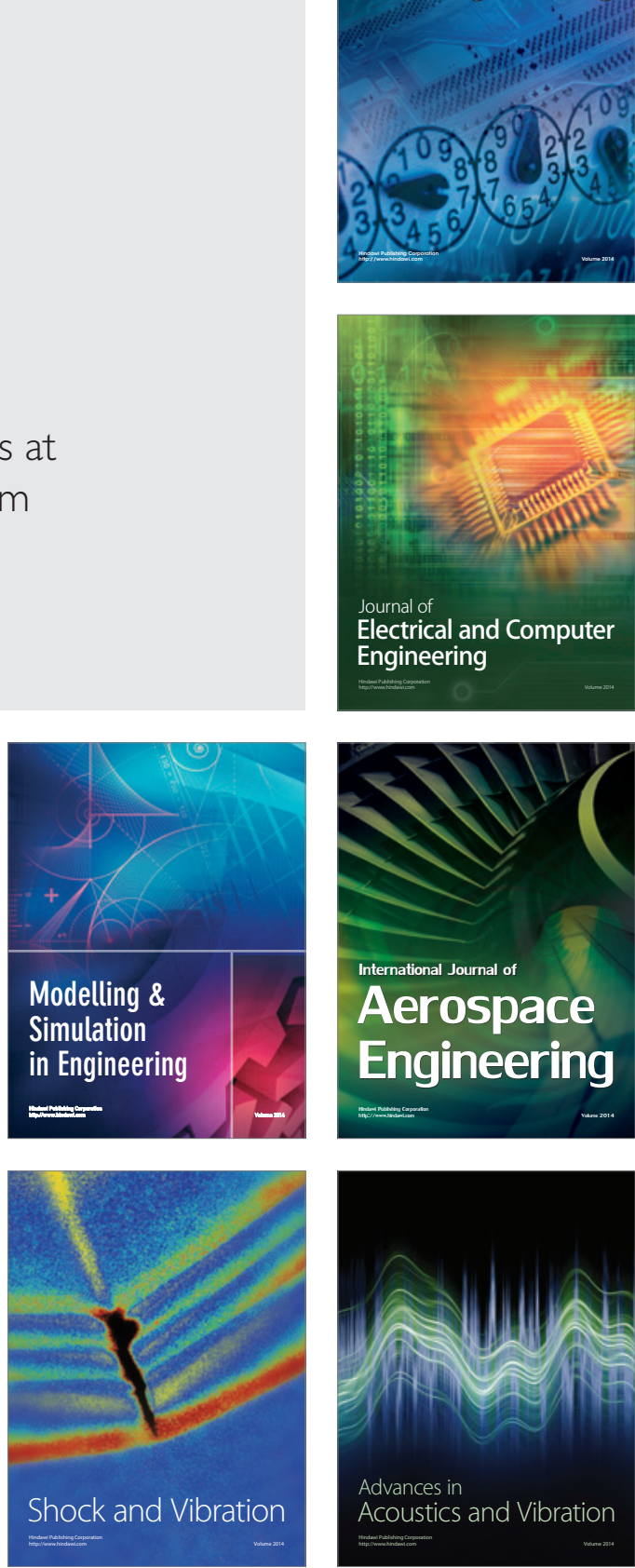